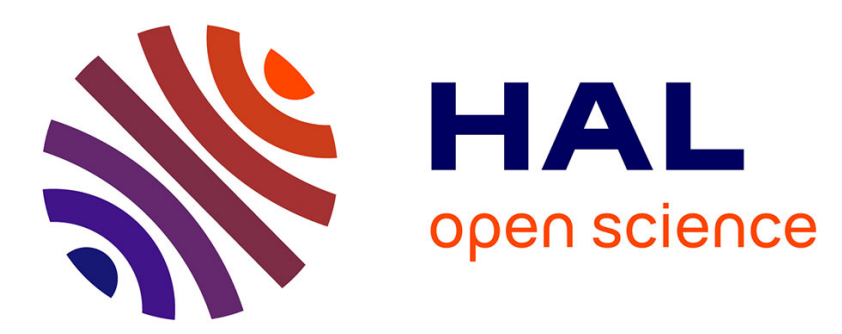

\title{
Récents développements de la recherche sur la génétique de Bacillus thuringiensis Berliner \\ Didier Lereclus
}

\section{To cite this version:}

Didier Lereclus. Récents développements de la recherche sur la génétique de Bacillus thuringiensis Berliner. Agronomie, 1984, 4 (3), pp.269-278. hal-00884633

\section{HAL Id: hal-00884633 \\ https://hal.science/hal-00884633}

Submitted on 1 Jan 1984

HAL is a multi-disciplinary open access archive for the deposit and dissemination of scientific research documents, whether they are published or not. The documents may come from teaching and research institutions in France or abroad, or from public or private research centers.
L'archive ouverte pluridisciplinaire HAL, est destinée au dépôt et à la diffusion de documents scientifiques de niveau recherche, publiés ou non, émanant des établissements d'enseignement et de recherche français ou étrangers, des laboratoires publics ou privés. 


\title{
Récents développements de la recherche sur la génétique de Bacillus thuringiensis Berliner
}

\author{
Didier LERECLUS \\ Institut Pasteur, Laboratoire de Biochimie microbienne, 28, rue du Docteur-Roux, F 75724 Paris \\ I.N.R.A., Station de Recherches de Lutte biologique, La Minière, F 78280 Guyancourt
}

Bacillus thuringiensis est une bactérie gram-positive ayant la particularité de synthétiser un cristal protéique cntomopathogène lors du processus de sporulation.

Les aspects génétiques de cet organisme procaryote ont été abordés par différentes voies : étude de l'ADN extrachromosomique, clonage du gène codant pour le cristal protéique, élaboration d'un système de transfert d'ADN.

La plupart des souches de $B$. thuringiensis cxaminćes possèdent des plasmides dont les masses moléculaircs sont comprises entre 1,5 ct 180 mégadaltons (Md). Certains plasmides sont conservés au travers des différents sérotypes de $B$. thuringiensis et peuvent être répartis en 2 catégorics de taille (inféricure et supćrieure à $15 \mathrm{Md}$ ) n'ayant pas d'homologie entre elles. Le gène codant pour le cristal protéique est généralement porté par un plasmide de masse moléculaire supéricure ou ćgale à $30 \mathrm{Md}$. Néanmoins, il existe dans certaincs souches d'autres situations concernant la localisation de ce gène. Le gène du cristal peut s'exprimer chez Escherichia coli et Bacillus subtilis où la quantité de protéines toxiques produites représente $10 \mathrm{p} .100 \mathrm{de} c c$ qui est obtenu chez B. thuringiensis.

Trois systèmes de transfert d'ADN ont été mis en évidence chez B. thuringiensis: la transduction, la transformation ct un mécanisme assimilable à la conjugaison. Ce dernier système s'est révélé particulièrement efficace pour transférer des plasmides et done pour étudier leur fonction dans la cellule.

Mots clés additionnels : Protéine entomopathogène, plasmides, recombinaison génétique in vitro, transfert d'ADN, transduction.

The gram-positive bacterium, Bacillus thuringiensis, produces an insecticidal proteinaceous crystal which is synthesized during the sporulation process.

The genetics of this prokaryotic organism was approached through different lines of research, namcly: characterization of the extrachromosomal DNA, cloning of the crystal protein gene and claboration of a DNA transfer system.

Most $B$. thuringiensis strains examined possessed plasmids with molecular weights ranging from 1.5 to 180 megadaltons (Md). Some plasmids were conserved among different serotypes of $B$. thuringiensis and could be distributed into 2 size classes (inferior and superior to $15 \mathrm{Md}$ ) having no homology between them. Generally, the gene coding for the crystal protein was carried by a plasmid with a molecular weight superior or equal to $30 \mathrm{Md}$. Nevertheless, various combinations were found regarding the localisation of this gene. The crystal gene was expressed in Escherichia coli and Bacillus subtilis., in these cases the amount of insecticidal proteins produced represented $10 \%$ of that produced by $B$. thuringiensis.

Three DNA transfer systems proved to be effective in $B$. thuringiensis, they were : transduction, transformation and a conjugation-like mechanism. This last system was highly efficient in transferring plasmids and could thus be used to study their function in the cell.

Additional key words : Insecticidal protein, plasmids, genetic manipulation, DNA transfer, transduction.

\section{INTRODUCTION}

Depuis une quinzaine d'années l'activité entomopathogène de Bacillus thuringiensis Berliner est bien connue (ANGUS \& NORRIS, 1968 ; LECADET, 1970) et permet déjà de combattre sélectivement un nombre limité de lépidoptères défoliateurs dont la nocuité se manifeste, par exemple (MARTOURET, 1978):

— en grandes cultures (pyrale du maïs, pyrale du riz...),

- en cultures maraîchères (piérides du chou, teigne des crucifères...),

- en viticulture (tordeuse de la grappe, Arctia caja L...),

- en arboriculture fruitière (cheimatobie, teigne de l'olivier, écaille fileuse, bombyx cul brun, bombyx livrée...), 
- ou encorc en forêt (tordeuse verte, bombyx dispar, chenilles processionnaires du chêne et du pin, tordeuse du mélèze).

Cette potentialité reste toutefois peu utiliséc en agriculture, en comparaison des insecticides chimiques. Aujourd'hui, alors que des phénomènes de résistance aux insecticides chimiques sont souvent observés, les possibilités, anciennes et nouvelles, offertes par B. thuringiensis suscitent un regain d'intérêt. La protéine toxique $\delta$-cndotoxinc, produitc par cette bactérie lors du processus de sporulation, ne semble pas, en effet, induire de phénomènes de résistance chez les insectes et, par ailleurs, il a été récemment montré que le spectre d'action de cette toxine n'est pas sculcment limité aux lépidoptères mais qu'il s'étend aussi à certains diptères (de BARJAC, 1978). De plus, les technicues de recombinaison génétique in vitro et de mutagenc̀se dirigéc permettent maintenant d'envisager l'élargissement de son spectre d'activité insecticide à d'autres espèces d'insectes nuisibles, qu'clles soicnt hématophages et vectrices de maladies parasitaires ou bien phytophages et déprédatrices de certaines de nos productions agricoles.

La synthèse clu cristal, qui apparaît dès le stade II de la sporogenèse, s'effectue à partir d'un ARN messager relativement stable (GLATRON \& RAPOPORT, 1972) transcrit préférenticllement par l'unc des ARN polymérases spécifiques de la sporulation (KLIER et al., 1978). La structure cristalline de l'inclusion résulte de l'assemblage de sousunités protéiques identiques ou protoxines de 130000 daltons (GlatRoN et al., 1972; Bulla et al., 1977). Lc cristal protéique digéré à $\mathrm{pH}$ alcalin par les protéases de l'insecte est transformé en toxines polypeptidiques actives de 68000 daltons (LECADET \& MARTOURET, 1962, 1967; BULla et al., 1979 ; LUTHY \& EBERSOLD. 1981). Ces résultats ont été démontrés pour la plupart des souches de $B$. thuringiensis; cependant, chez la souche israelensis, sćrotype 14, qui est active contre certains diptères, la protoxine: serait de taille inféricure; un composant majoritaire de 26000 daltons a en effet été trouvé chez cette souche (TYRELL et al., 1981).

Il existe aussi une importante documentation sur les proprićtés biochimiques d'une autre toxine de $B$. thuringiensis: la $\beta$-exotoxine ou thuringiensine (LECADET \& DE BARJAC, 1981). Cette toxine thermostable est de nature nucléotidique; sa structure moléculaire ainsi que son mécanisme d'action sur l'ARN polymérase des eucaryotes ont été clairement démontrés (FARKAS et al., 1969 ; SEBESTA \& HORSKA, 1970). Compte tenu de son effet inhibiteur sur la synthèse des ARN, le spectre d'action de la $\beta$-exotoxine cst moins spécifique que celui du cristal protéique; il touche en effet de nombreux insectes appartenant à plusicurs ordres. De ce fait, son application agronomique, plus difficilement contrôlable, n'est pas autoriséc à l'heure actuelle.

Ces connaissances biochimiques sur les différentes toxines de $B$. thuringiensis ont largement devancé les recherches sur la génétique et, en particulier, celles concernant les gènes impliqués dans la synthèse de ces toxines. Ce retard est principalement dû au fait que les 3 principaux systèmes d'investigation génétique: la transduction, la transformation et la conjugaison n'ont commencé à être ćtudićs que depuis 5 ans chez $B$. thuringiensis (LECADET et al., 1980 ; MARTIN et al., 1981 ; GonZalez \& CARLTON, 1982).

Parmi les différentes voies qui ont donc permis l'approche génétique de cette bactérie, nous présentons ici certains des résultats significatifs de l'évolution de nos connaissances sur les aspects génétiques de $B$. thuringiensis.
II. CARACTÉRISATION PHYSIQUE DES PLASMIDES DE B. THURINGIENSIS

Les travaux concernant l'ADN extrachromosomique de $B$. thuringiensis ont constitué la première approche d'une étude génétique chez cet organisme, d'une part parce qu'ils ne nécessitaient pas d'autres connaissances d'ordre génétique, et d'autre part en raison du caractère non essentiel, pour la bactéric, des toxines entomopathogènes produites lors de la sporulation, ce dernier point dénotant la possibilité d'une localisation plasmidique des gènes responsables de la synthèse de ces toxines.

Les premiers résultats qui ont indiqué la présence d'ADN extrachromosomique dans plusicurs souches de B. thuringiensis (DEBABOV et al., 1977 ; STAHLY et al., 1978) ont aussi montré qu'il existait une relation entre la $\delta$-cndotoxine et les plasmides. Les auteurs remarquaient en effet que la disparition du cristal protéique coïncidait avec la perte de la totalité de l'ADN extrachromosomique dans ces bactérics.

La présence de molécules d'ADN extrachromosomique a, par la suite, été révélée dans la plupart des souches de B. thuringiensis (GONZALEZ \& CARLTON, 1980 ; LECADET et al., 1981) ; leur structure hélicoïdale, caractéristique des plasmides, a aussi pu être observée en microscopie électronique par J. RIBIER (LERECLUS, 1981).

Des examens plus systématiques des contenus plasmidiques de plusicurs souches, appartenant à des sérotypes différents, ont conduit à faire une caractérisation physique des plasmides en fonction de leur masse moléculaire (GONZALEZ et al., 1981; LERECLUS et al., 1982). Lc tableau 1 présente une grande partic des résultats obtenus et fait apparaitre la complexité et la diversité des schémas plasmidiques mis en ćvidence par ćlectrophorèse $\mathrm{cn}$ gel d'agarose ou par microscopic ćlcctronique. Il ressort en effet que presque toutes les souches possèdent des plasmides dont les masses moléculaires sont comprises entre 1,5 et $180 \mathrm{Md}$. On peut remarquer que toutes ces souches, dont la caractéristique commune est la synthèse d'un cristal toxique, ont des constituants extrachromosomiques de taille ćlcvéc (supéricure à $30 \mathrm{Md}$ ). Seulc la souche entomocidus du sérotype 6 ne semble pas posséder de plasmides, bien qu'clle soit aussi productrice de cristal.

En ce qui concerne la relation des plasmides avec le cristal protéique, GonZaLEz et al. (1981) ont recherché de façon systématique si la perte d'un plasmide entraînait le phénotype $\mathrm{Cry}^{-}$(absence de cristal). Cette étude a été réaliséc, d'unc part avec des mutants spontanćs $\mathrm{Cry}^{-}$, et d'autre part après traitement des souches sauvages par le SDS (sodium dodecyl sulfate), suivant la méthode décrite par BERNHARD et al. (1978), afin de provoquer la perte de plasmides. Ce type d'investigation a clairement montré que, dans le cas de 2 souches : thuringiensis HD2 (sérotype 1) et kurstaki HD73 (sérotype 3), les plasmides de $75 \mathrm{Md}$ et de $50 \mathrm{Md}$ étaient respectivement absents des mutants $\mathrm{Cry}^{-}$.

Une étude des homologies moléculaires existant entre ces différents plasmides a montré que la diversité physique des contenus extrachromosomiques n'avait pas son équivalent au niveau moléculaire. Deux groupes de taille (supérieure ou inférieure à $15 \mathrm{Md}$ ) ont en effet pu être distingućs. Ces 2 groupes ne présentent que très peu d'homologies entre eux, alors qu'à l'intérieur de chacun d'eux il existe une conservation, particlle ou totale, de certains plasmides au travers des différents sérotypes (LERECLUS et al., 1982). On peut noter, comme exemple, le cas où les plasmides de la souche subtoxicus (sérotype 6) sont marqués au ${ }^{32} \mathrm{P}$ et utilisés comme sonde radioactive lors d'hybridations moléculaires réalisées avec des plasmides provenant d'autres 
TABLEAU 1

Contenus plasmidiques de 19 souches de $\mathrm{B}$. thuringicnsis productrices de la $\delta$-endotoxine. Plasmid content of 19 crystalliferous strains of $\mathrm{B}$. thuringicnsis.

\begin{tabular}{|c|c|c|c|}
\hline H. sérotype & Souches ${ }^{b}$ & $\begin{array}{l}\text { Nombre de } \\
\text { plasmides }\end{array}$ & $\begin{array}{l}\text { Plasmides observés par électrophorèse en gel d'agarose } \\
\text { ou par microscopie électronique }{ }^{\mathbb{2}} \text { (masses moléculaires exprimées en } \mathrm{Md} \text { ) }\end{array}$ \\
\hline 1 & berliner 1715 & 17 & 3,$9 ; 5,4 ; 7,5 ; 9,5 ; 15 ; 17 ; 21 ; 25 ; 28 ; 39 ; 42 ; 51 ; 60 ; 77 ; 100 ; 120 ; 180$ \\
\hline 1 & berliner 22105 (type) & 7 & 5,$4 ; 6.2 ; 7,5 ;(15) ; 33 ; 56 ; 58$ \\
\hline 1 & thuringiensis $\mathrm{HD} 2$ & 9 & $5.2: 6.2: 7.2 ; 7.6: 32 ; 37 ; 54: 57: 75:-150$ \\
\hline $3 \mathrm{a}$ & alesti HD4 & 10 & $4.6: 4.9 ; 5,4 ; 6, \overline{6,2} ; 6,4: 35 ; 37: 39: 46 ; 105$ \\
\hline $3 a, 3 b$ & kurstaki (typc) & 5 & 5,$2 ; 5,6 ; 7,8 ;(10,5) ; 54$ \\
\hline $3 a, 3 b$ & kurstaki HD1 & 8 & 1,$5 ; 5,2 ; 5,6 ; 9,3 ;(10) ; 30 ; 47 ; 54$ \\
\hline $3 a, 3 b$ & kurstaki HD73 & 6 & 4,$9 ; 5,2 ; 5,4 ; 7,5 ; 50 ; 50$ \\
\hline $3 a, 3 b$ & kurstaki HD 73.6 & 6 & $4.9 ; 5.2 ; 6.7 ; 7.5 ; 43: 50$ \\
\hline $4 a, 4 b$ & sotto (type) & 3 & $5.2 ; 39: 43$ \\
\hline $4 a, 4 b$ & dendrolimus (typc) & 4 & $33 ; 37 ; 66 ; 73$ \\
\hline $5 \mathrm{a}, 5 \mathrm{~b}$ & galleriae $\mathrm{G} 1$ & 5 & 6,$3 ; 8,6 ; 20 ; 51 ; 74$ \\
\hline $5 a, 5 b$ & galleriae HD8 & 4 & 5,$2 ; 8.7 ; 10,3 ; \sim 130$ \\
\hline 6 & entomocidus (type) & 0 & \\
\hline 6 & subtoxicus (typc) & 2 & $52: 56$ \\
\hline 7 & aizawaï (typc) & 8 & 3,$9 ; 5,4 ; 5,6 ; 7 ; 8,5 ; 30 ; 35 ;(60)$ \\
\hline 7 & aizawai 7.21 & 8 & $4 ; 6.6 ; 7.5 ; 8,9 ; 15 ; 18 ; 40 ; 50$ \\
\hline 9. & tolworthi (typc) & 6 & $5.2 ; 7.6 ; 8 ;(16) ; 28 ; 45$ \\
\hline 14 & israelensis HD567 & 9 & $3.2: 4.1: 4.9: \sim 10: 10.5: 66: 72: \sim 100: \sim 130$ \\
\hline 14 & israelensis (type) & .6 & $4 ; 4,5 ; 5,3 ;(10) ; 68 ; 72$ \\
\hline
\end{tabular}

a : Sérotype flagellaire, en accord avec la classification de de BARIAC (1981).

b: Les souches marquées «type "sont répertoriées dans la classification de de BARIAC \& BONNEFOI (1968).

$\mathrm{c}$ : Les masses des plasmides des souches berliner 1715, thuringiensis HD2, dendrolimus, galleriae G1 et subtoxicus ont ćté déterminées par la mesure, en microscopie électronique, du contour de leur forme circulaire ouverte (exception faite du plasmide de $150 \mathrm{Md}$ de la souche thuringiensis $\mathrm{HD} 2$ ). Ces plasmides ont ćté utilisés comme marqucurs en gel d'agarose pour détermincr la masse moléculairc des autres plasmides de $B$. thuringiensis.

- Les masses entre parenthèses correspondent à des molécules d'ADN extrachromosomique présentes en faible quantité et qui ne sont pas détectées de façon systématique.

- Les masses soulignées sont celles de plasmides isolés principalement sous la forme circulaire ouverte. Dans le cas du plasmide de 7,6 Md de la souche HD2 aucune forme hélicoïdale (superenroulée) n'a été observéc. Le constituant de $10 \mathrm{Md}$ de la souche israelensis HD567 n'est présent que sous la forme linéaire.

N.B. : Il s'agit là d'une caractérisation physique des différentes espèces de plasmides dont certaincs d'entre elles peuvent représenter les formes dimériques d'espèces de masses moléculaires 2 fois inféricurcs. D'après GonZAlez et al. (1981), GONZALFZ \& CARLTON (1982), LERECLUS et al. (1982), LERECLUS et al. (1983).

a: Flagellar serotype according to de BARJAC s classification (1981).

b: Strains marked "type" are indexed in the classification of de BARJAC \& BONVEFOI (1968).

$c$ : Masses of plasmids from berliner 1715, thuringiensis $\mathrm{HD2}$, dendrolimus, galicriae $G 1$ and subtoxicus strains were determined by contour length measurements of their open circular form by electron microscopy (with the exception of the $150 \mathrm{Md}$ plasmid from the thuringiensis $H D 2$ strain). These plasmids were used as molecular weight standards on agarose gels to obtain molecular weights for the other

B. thuringicnsis plasmids.

- Masses in parentheses correspond to extrachromosomal DNA present in minor amounts and not detected systematically.

- Underlined masses are those of plasmids isolated predominantly in the open circular form. In the case of the $7.6 \mathrm{Md}$ HD2 plasmid, no supercoiled DNA has ever been detected. The $10 \mathrm{Md}$ plasmid from the israclensis $\mathrm{HD} 567$ strain is only present under linear form.

N.B. : This table shows a physical characterization of different species of plasmids; perhaps some of them may represent the dimeric forms of species with lower molecular weights.

souches. Les résultats obtenus dans ce type d'expérience montrent que ces 2 plasmides de 52 et $56 \mathrm{Md}$ hybrident sculement avec les plasmides de masse moléculaire élevéc (comprise entre 40 ct $70 \mathrm{Md}$ ) appartenant aux sćrotypes 1 . $3,4,5$ et 9 . Dans un seul cas, nous avons pu observer une homologie entre cette sonde radioactive et un constituant de taille nettement inféricure, le plasmide de $9,5 \mathrm{Md}$ de la souche berliner 1715 (sérotype 1). Ces résultats suggèrent donc clairement qu'il existe des séquences d'ADN extrachromosomique communes à différents sérotypes de $B$. thuringiensis ct que l'on peut ainsi considérer comme étant caractéristiques de l'cspèce. Un autre résultat récent semble par ailleurs confirmer cette hypothèse : il s'agit de l'isolement d'une séquence d'ADN de $3 \mathrm{Md}$ provenant du plasmide de $54 \mathrm{Md}$ de la souche kurstaki type. Cet isolement a été obtenu par insertion in vivo du fragment de $3 \mathrm{Md}$ sur le plasmide conjugatif pAM $\beta 1$ (LERECLUS et al., 1983). Il s'est avéré par la suite que cette séquence d'ADN de 3 Md était présente dans plusicurs souches dont la souche berliner 1715 où elle était localisée à la fois sur les plasmides de $9.5 \mathrm{et} \mathrm{de}$ $42 \mathrm{Md}$.

\section{LOCALISATION, CLONAGE ET EXPRESSION DES GËNES CODANT POUR LE CRISTAL PROTÉIQUE}

En même temps que l'étude des plasmides de $B$. thuringiensis, plusicurs travaux ont été réalisés sur le gène de structure du cristal protéique. Les recherches ont abouti à cloner le gène, par recombinaison génétique in vitro, dans des micro-organismes génćtiquement micux connus : Escherichia coli Castellani \& Chalmers et Bacillus subtilis Cohn. Chronologiquement, les premiers travaux menés sur ce sujet ont consisté à construire une banque de clones représentant l'ensemble du génome (ADN chromosomique 
et extrachromosomique) de $B$. thuringiensis, souche berliner 1715 (KLIER et al., 1979). A partir de la totalité des clones, dont le plasmide pBR322 constituait le vecteur, ces auteurs ont isolé une sćquence d'ADN de 4 kilobases ( $\mathrm{kb}$ ) hybridant avec un ARN messager de $26 \mathrm{~S}$, spécifique de la sporulation et dont la taille était suffisante pour coder une protéine d'environ 150000 daltons.

Il s'est avéré que, chez $E$. coli et $B$. subtilis, ce fragment d'ADN, d'origine chromosomique ou, comme le suggèrent KRONSTAD et al. (1983), provenant d'un plasmide de masse moléculaire très élevéc ( $>150 \mathrm{Md})$, codait bien pour un ARN messager de 15 à $26 \mathrm{~S}$ qui n'était toutefois pas traduit en protéinc. Le fragment de $4 \mathrm{~kb}$ a néanmoins pu cetre utilisé pour localiser, par hybridation moléculaire, une séquence d'ADN présentant avec lui d'importantes homologies sur un plasmide de $40 \mathrm{Md}$ de la même souche berliner 1715 (KLIER et al., 1982). Cette séquence d'ADN plasmidique contenait en réalité le gène actif du cristal protéique puisque, clonée chez E. coli, les mêmes auteurs ont observé qu'clle était responsable de la synthèse d'une protéine toxique contre les larves de lépidoptères et présentant les mêmes caractéristiques antigéniques que la $\delta$-endotoxine. Il résultait donc de cette étude que, dans la souche berliner 1715,2 séquences homologues du gène du cristal protéique ćtaient présentes : d'une part, sur le chromosome ou sur un plasmide de $150 \mathrm{Md}$ et, d'autre part, sur un plasmide de $40 \mathrm{Md}$.

La synthèse des sous-unités protéiques constitutives du cristal a été sculement obtenue à partir du gène d'origine plasmidique: chez E. coli, dans des cellules en phase végétative et, chez $B$. subtilis, dans les cellules engagées dans le processus de sporulation. Ce dernicr point indique que l'expression du gène est vraisemblablement soumise à un mécanisme de régulation identique chez $B$. thuringiensis et $B$. subtilis. Par ailleurs, les autcurs de ce travail estiment que la quantité de protéine toxique produite dans $E$. coli $\mathrm{et}$ $B$. subtilis représente environ 10 p. 100 de ce qui est généralement obtenu chez $B$. thuringiensis. Ce pourcentage relativement faible pourrait être à l'origine de l'absence du phénomène de cristallisation des sous-unités protéiques dans ces 2 organismes.

Parallèlement à l'ćtude précédente effectuée sur la souche berliner 1715, 2 autres équipes ont réalisé le clonage des gènes du cristal d'une souche kurstaki. Deux types de méthodologic, utilisation du vecteur plasmidique $\mathrm{pBR} 322$ (SCHNEPF \& WhITELEY, 1981) et du phage Charon 4A (HELD et al., 1982), ont respectivement abouti au clonage du gène porté par un plasmide et d'un gène présumé d'origine chromosomique. Dans les 2 cas, les clones recombinants obtenus chez E. coli ont été détectés pour leur production de protoxine par radioimmunologie. Donc, dans la souche kurstaki, il y a là encorc une double localisation du gène mais, à la différence de la $1^{\text {rc }}$ expérience, les 2 gènes auraient la propriété de s'exprimer chez E. coli.

L'utilisation des gènes clonés a par ailleurs permis de déterminer précisćment, par hybridation moléculaire, la localisation des gènes du cristal dans plusicurs sérotypes de B. thuringiensis. La figure 1 représente les contenus plasmidiques de 4 souches différentes, où sont indiqués les plasmides porteurs du gène du cristal. Dans le cas de ces 4 souches, il s'agit d'un plasmide de masse moléculaire comprise entre 40 et $60 \mathrm{Md}$ (KLIER et al., 1982). De plus, les hybridations moléculaires avec les gènes clonés ont mis en évidence les diverses situations relatives à la localisation plasmidique et/ou chromosomique du gène du cristal. Ces résultats seront rappelés ultéricurement lors de la discussion (tabl. 4).

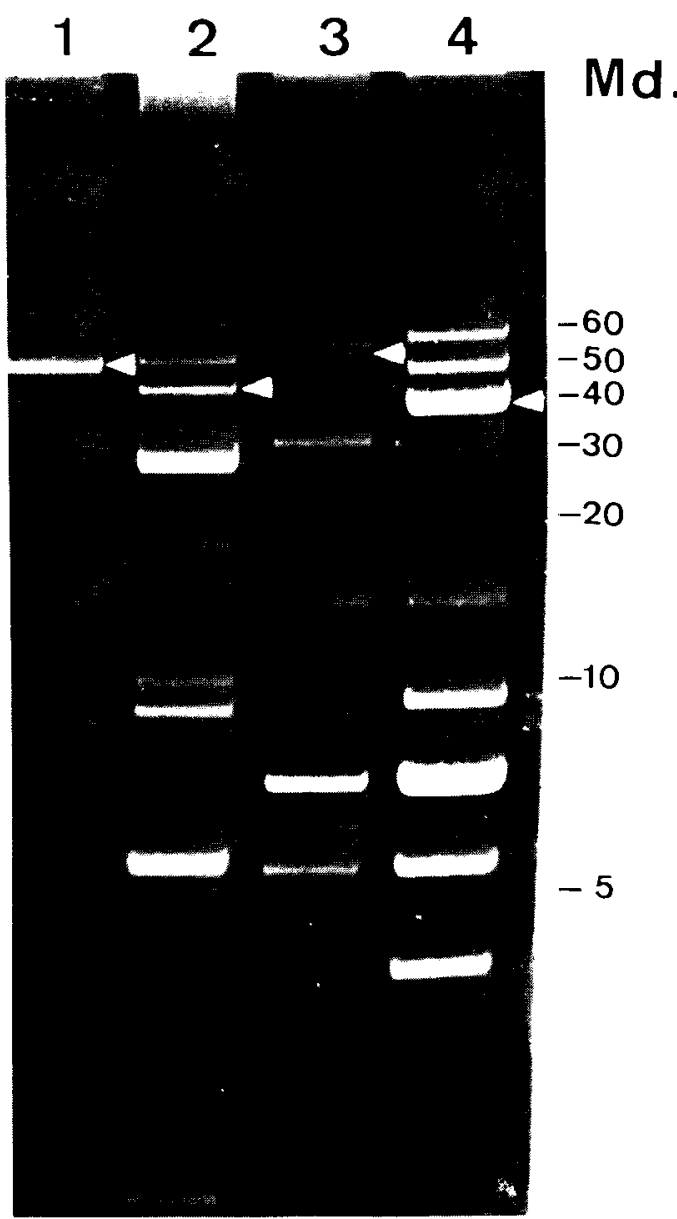

Figure 1

Localisation du gène plasmidique codant pour la $\delta$-endotoxine dans 4 souches de $\mathrm{B}$. thuringiensis.

Photographie d'un gel d'agarose, après coloration au bromure d'éthidium, contenant l'ensemble des plasmides de 4 souches de $\mathrm{B}$. thuringiensis ; $1=$ subtoxicus (type) $; 2=$ kurstaki $H D l ; 3=$ berliner $22105 ; 4=$ berliner 1715 .

Les flèches indiquent les plasmides porteurs du gène du cristal protéique. Md représente l'échelle des masses moléculaires en mégadaltons.

Location of the 8 -endotoxin plasmid gene in $4 \mathrm{~B}$. thuringicnsis strains.

Agarose gel of total plasmids, from $4 \mathrm{~B}$. thuringiensis strains, stained with ethidium bromide: 1 = subtoxicus (wild type) ; 2 = kurstaki $\mathrm{HDI} ; 3=$ berliner $22105 ; 4=$ berliner 1715 .

Arrows indicate the plasmids harbouring the crystal protein gene. $M d$ represents the scale of molecular weights in megadaltons.

Finalement, le clonage des gènes codant pour le cristal protéique a récemment permis d'établir la séquence d'ADN des promoteurs des gènes d'origine plasmidique (WONG et al., 1983) et chromosomique (KLIER et al., 1983). Dans les 2 cas une forte affinité a pu être observée entre ces promoteurs et l'ARN polymérase - forme II - spécifique de la sporulation (KLIER et al., 1978). De plus, certaines similitudes ont pu ĉtre dégagées entre le promoteur du gène du cristal d'origine plasmidique et les promoteurs des gènes de sporulation de B. subtilis. Ces résultats suggèrent donc que l'expression des gènes responsables de la synthèse du cristal protéique et l'expression des gènes chromosomiques de sporulation sont soumises à des mécanismes de régulation proches, sinon identiques. 


\section{TRANSFERT D'ADN DANS B. THURINGIENSIS}

D'une façon générale, bien que les techniques de recombinaison génétique in vitro offrent la possibilité d'isoler et d'étudier l'expression de certains gènes chez des organismes autres que celui d'origine, il est encore difficile d'envisager l'étude génétique approfondie d'une espèce bactérienne sans disposer des moyens classiques permettant le transfert d'ADN à l'intéricur même de cette espèce. C'est pourquoi, depuis quelques années, de nombreuses recherches ont été entreprises sur les systèmes de transformation, conjugaison et transduction chez $B$. thuringiensis.

\section{A. Transformation}

Aucun système de transformation sur les cellules entières et faisant intervenir un état de compétence n'a été mentionné chez $B$. thuringiensis. En revanche, plusicurs tentatives de transformation des protoplastes par des plasmides en présence de polyéthylène glycol ont été effectuées chez $B$. thuringiensis suivant la méthode décrite par CHANG \& COHEN (1979). Les expériences de transformation ont été réalisées avec 3 plasmides différents : les plasmides pBC16 de Bacillus cereus Fr. and Fr. (AlikHANIAN et al., 1981), pUB110 (MITEVA et al., 1981) et pC194 (MARTIN et al., 1981) provenant de Staphylococcus aureus Rosenb. L'cxpression de ces plasmides a pu être obtenue dans $B$. thuringiensis, mais les fréquences de transformation restaient relativement faibles dans la plupart des cas (environ $10^{-6}$ transformant par ccllule). Deux autres caractéristiques rendaicnt cette méthodologie peu fiable; tout d'abord l'importante quantité d'ADN requise ( $\geqslant 1 \mu \mathrm{g}$ pour chaque expérience de transformation) et ensuite le fait que 2 souches seulement se sont révélées susceptibles d'être transformées, une souche galleriae appartenant au sérotype 5 et unc souche dérivéc de la varićté kurstaki Hol (sérotype 3).

On peut supposer que le caractère exceptionnel de la transformation de $B$. thuringiensis soit en partic dû aux conditions de régénération de la paroi bactérienne ne permettant qu'une réversion peu efficace et aléatoire des protoplastes en formes bacillaires viables (LERECLUS, 1981).

\section{B. Conjugaison}

Un phénomène assimilable à la conjugaison a été réccmment mis en évidence entre différentes souches de $B$. thuringiensis par GONZALEZ \& CARLTON (1982). Bicn que dans la suite du texte nous appelions ce phénomène "conjugaison ", quelque réserve doit être faite quant à sa définition. Il s'agit d'un mécanisme nécessitant le contact cellulaire de 2 populations bactériennes en croissance dans un milicu liquide et dont le résultat peut s'exprimer par l'observation d'un transfert de plasmides. Nous ne savons pas si ce mécanisme présente un caractère actif comme la conjugaison chez les bactéries Gram-nćgatives. Toutefois, l'insensibilité du phénomène à la présence de désoxyribonucléase implique que le transfert se produit dans des conditions où l'ADN est protégé, tout au moins partiellement, du milicu extracellulaire. GONZALEZ \& CARLTON ont par ailleurs montré que le processus de transfert ne fait pas intervenir de particules phagiques.

Le tableau 2 résume quelques résultats obtenus par cette méthode de conjugaison. Il apparaît que certains plasmides sont transférables à des fréquences ćlcvées (50 p. 100 des cellules réceptrices) alors que d'autres ne le sont pas, tout au moins avec de telles fréquences. Ces différences peuvent exprimer des phénomènes d'incompatibilité entre plasmides, voire même entre souches; pour une souche réceptrice donnée, n'importe quelle autre souche ne pourrait en effet être utiliséc comme donneuse. Ces expériences ont permis à GONZALEZ et al. (1982) de faire 2 constatations concernant la fonction de plusicurs plasmides:

- l'introduction du plasmide de $43 \mathrm{Md}$ de la souche HD 73.6 entraîne une modification de l'aspect colonial du transconjugant par rapport à la souche réceptrice ;

- Ic transfert de certains plasmides (par exemple lc plasmide de $50 \mathrm{Md}$ de la souche $\mathrm{HD}$ 73.6) rétablit la production de cristal chez les transconjugants obtenus à partir de bactéries réceptrices $\mathrm{Cry}^{-}$(tabl. 2). Ces cristaux présentant la même spécificité antigénique que ceux de la souche donneuse, il paraît donc évident que le ou les gènes codant pour la $\delta$-endotoxinc sont bien portés par le plasmide transféré.

Une $2^{c}$ approche du système de conjugaison a été abordée au laboratoire où nous avons essayé de pallier l'absence de marqueur permettant de sélectionner les transconjugants. Tous les plasmides n'étant pas transmissibles avec des fréquences ćlcvées, il ćtait nécessaire de disposer d'un vecteur portcur d'un marqueur sélectif afin de suivre par coconjugaison le transfert des plasmides cryptiques de $B$. thuringiensis.

Dans ce but, le plasmide pAM $\beta 1$ de Streptococcus faecalis Andr. \& Horder (CLEWELl et al., 1974) a ćté utilisé lors d'expériences de conjugaison intraspécifique dans $B$. thuringiensis. Ce plasmide porte un gène conférant la résistance à l'érythromycine et possède des propriétés mobilisatrices d'ADN plasmidique chez $S$. faecalis. Le plasmide $\mathrm{pAM} \beta 1$ a tout d'abord été introduit dans différents sérotypes de $B$. thuringiensis par conjugaison intergénérique avec $S$. faecalis puis utilisé par la suite pour suivre le processus de conjugajson entre souches de $B$. thuringiensis. La sélection des transconjugants pour l'acquisition de la résistance à l'érythromycine a ainsi permis de déceler le transfert de plasmides cryptiques résidents de B. thuringiensis (tabl. 2) (LERECLUS et al., 1983).

II apparaît que, dans le cas de la souche subtoxicus, le passage d'un plasmide de 52 ou $56 \mathrm{Md}$ est observé uniquement parmi les transconjugants sélectionnćs pour la résistance à l'érythromycine et ayant donc reçu le plasmide pAM $\beta 1$. Lors d'expériences de conjugaison réalisées avec d'autres souches, cette méthode s'est aussi révéléc efficace pour sćlectionner, par le biais de pAM $\beta 1$, le transfert de plasmides n'étant pas auto-transmissible, tout au moins avec des fréquences élevées, chez $B$. thuringiensis. Néanmoins, il est encore trop tôt pour conclure que le plasmide pAM $\beta 1$ Bexprime, chez $B$. thuringiensis, la proprićté de mobiliser l'ADN plasmidique durant la conjugaison.

\section{Transduction}

Décrit pour la première fois par THORNE (1978), le système de transduction chez $B$. thuringiensis a été utilisć par plusicurs équipes pour localiser des marqueurs chromosomiques et ébaucher ainsi plusicurs groupes de liaison chez différentes souches de cet organisme (THORNE, 1978; PERLAK et al., 1979 ; LECADET et al., 1980 ; LANDEN et al., 1981). Deux phages ont été principalement utilisés lors de ces expériences: le phage CP54 de B. cereus et le phage $\Phi 63$ de $B$. thuringiensis, tous deux isolés du sol. Ces 2 particules phagiques ont la potentialité de transférer de l'ADN par un mécanisme de transduction généralisée, avec des fréquences comprises entre $5.10^{-7}$ et $5.10^{-6}$ pour un 


\section{TABLEAU 2}

Transfert intraspécifique de plasmides dans $\mathrm{B}$. thuringicnsis. Intraspecific transfer of plasmids in $\mathrm{B}$. thuringiensis.

\begin{tabular}{|c|c|c|c|c|}
\hline Souches donneuses & $\begin{array}{l}\text { Souches réceptrices } \\
\text { résistantes à la } \\
\text { streptomycine }\left(\mathrm{Str}^{\mathrm{R}}\right)^{\mathrm{a}}\end{array}$ & $\begin{array}{l}\text { Plasmides } \\
\text { transmis }\end{array}$ & $\begin{array}{c}\text { Pourcentage } \\
\text { de transfert } \\
\text { observé parmi } \\
\text { les colonies } \\
\text { réceptrices } \\
\left(\mathrm{Str}^{\mathrm{R}}\right) \text { ou }\left(\mathrm{Str} \mathrm{Em}^{\mathrm{R}}\right)\end{array}$ & Références \\
\hline kurstaki HD 73-6 & thuringiensis HD2 cry & $\begin{array}{l}50 \mathrm{Md}^{\mathrm{b}} \\
43 \mathrm{Md}^{\circ} \\
6.7 \mathrm{Md} \\
4.9 \mathrm{Md}\end{array}$ & $\begin{array}{l}\sim 20 \% \\
-50 \%\end{array}$ & GONZALI: \& CARLTON (1982) \\
\hline kurstaki HD1 & thuringiensis HD2 $\mathrm{cry}^{-}$ & Aucun & & GONZALIZ \& CARLTON (1982) \\
\hline kurstaki (type) & kurstaki HD1 cry ${ }^{-} \mathrm{B}$ & $\begin{array}{l}5,6 \mathrm{Md} \\
5.2 \mathrm{Md}\end{array}$ & $\sim 50 \%$ & LERECLUS et al. (1983) \\
\hline subtoxicus (type) & kurstaki HDl cry $^{-}$B & Aucun & $<10 \%$ & LERFCLUS et al. (1983) \\
\hline subtoxicus $\mathrm{Em}^{\mathrm{R}}$ (pAM $\left.\beta 1\right)$ & kurstaki HDI cry ${ }^{-} \mathrm{B}$ & 52 ou $56 \mathrm{Md}$ & $>50 \%{ }^{d}$ & LERECLUS et al. (1983) \\
\hline
\end{tabular}

a : 11 s'agit dans tous les cas de mutants cristal ${ }^{-}$dérivés des souches $1 \mathrm{D} 2$ ou HD1.

b: Lintroduction de ce plasmide rétablit la production de cristal protéique.

c : L'introduction de ce plasmide modific l'aspect colonial de la souche réceptrice.

d: Le transfert est obscrvé parmi les transconjugants sélectionnés pour l'acquisition de la résistance à l’érythromycine (Em ${ }^{R}$ ) donc si le plasmide pAN $\beta 1$ présent dans la souche donneuse a aussi ćté transférć.

N.B. : Le transfert intraspécifique des plasmides cryptiques est observé par électrophorèse en gel dagarose du contenu extrachromosomique des souches réceptrices.

$a:$ In all cases the crystal mutants derived from the $\mathrm{HD} 2$ and $\mathrm{HD}_{\mathrm{D}} \mathrm{l}$ strains.

$b$ : The introduction of this plasmid restored the crystal protein production.

$c$ : The introduction of this plasmid induced a change in colony morphology of the recipient strain.

$d$ : The transfer was observed among the transconjugants after selection for the resistance to erythromycine (Em ${ }^{R}$ ) thus indicating that the pAm 1 plasmid present in the donor strain had been transfered.

N.B. : Intraspecific transmission of cryptic plasmid was observed by examining plasmid patterns of the recipient strains.

caractère donné. Un dérivé du phage CP54, désigné CP54Ber pour sa capacité à transduire la souche berliner 1715, a été plus particulièrement étudié (LlECADET et al., 1980). Il s'agit d'un phage à structure icosaćdrique dont le matćricl génétique est constituć par une molécule d'ADN linéaire d'environ $70 \mathrm{Md}$.

Le tableau 3 rassemble en 5 groupes de liaison un certain nombre de loci correspondant à des marqueurs d'auxotrophie ou de résistance à des antibiotiques. Il faut toutcfois remarquer que les travaux qui ont abouti à l'établissement de ces groupes ont été réalisés avec des souches de $B$. thuringiensis différentes; de ce fait, quelque ambiguité peut apparaître à l'intéricur et entre chaque groupe de liaison. Bien que ces résultats ne concernent pas un sérotype unique de référęnce, ils peuvent néanmoins constituer une base de travail pour une étude plus approfondic sur le déterminisme génétique de la sporulation et de la synthèse de la $\delta$ endotoxine, au niveau de l'ADN chromosomiquc. Dans cette perspective, le bactériophage TP13 isolé par PERLAK el al. (1979) sera peut-être un « outil » intéressant. Ce phage a en effet la capacité de convertir, à des fréquences élevées, un mutant oligosporogène $\mathrm{Cry}^{-}$d'unc souche sotto en $\mathrm{Sp}^{+} \mathrm{Cry}^{+}$. Un tel résultat peut être dû à la transduction d'un gène intervenant dans la synthèse de l'ARN polymérase spécifique de la sporulation et nécessaire à la synthèse du cristal protéique mais il peut aussi indiquer l'existence d'une liaison génétique entre un gène de sporulation et un gène de la $\delta$-endotoxine éventucllement localisé sur le chromosome de cette souche.

\section{DISCUSSION, PERSPECTIVES ET CONCLUSIONS}

Il apparaît immédiatement, lorsqu'on essaye de rassembler tous les résultats des travaux effectućs sur $B$. thuringiensis, que le nombre important de souches (plus de 900), regroupées en 19 sérotypes, présente le risque de provoquer une divergence des recherches si une même nomenclature n'est pas utilisćc pour désigner les souches étudiées.

Le grand nombre de souches peut constituer un ćlément intéressant dans le cadre de certaines études comme celles concernant l'ADN extrachromosomique, sa variabilité, sa dissémination au travers des différents sérotypes parfois relativement ćloignés au niveau écologique et finalement son mode de transmission entre souches; cn revanche il demande bcaucoup plus de rigucur lorsqu'il s'agit d'étudier l'ADN chromosomique. Il est en effet assez étonnant de constater que les expériences de transduction, dont l'objectif est de regrouper sur un même chromosome tous les marqueurs génétiques de $B$. thuringiensis, soient réalisćes sur des souches différentes et non pas uniquement sur les micux connues (berliner 1715 ct kurstaki HD1).

En ce qui concerne l'étude des plasmides de $B$. thuringiensis, si l'absence d'une nomenclature uniforme des variétés ne facilite pas la comparaison entre les souches, les résultats obtenus par les différents laboratoires ont néanmoins pu fournir des donnécs comparables et complémentaires. Il ressort en effet du tableau 1, outre l'importante diversité de plasmides, qu'à l'intérieur d'un même sérotype il existe une relative similitude des plasmides en fonction de 
TABLEAU 3

Groupes de liaison de marqueurs chromosomiques établis par cotransduction dans $\mathrm{B}$. thuringiensis.

Linkage groups obtained by cotransduction of chromosomal markers in $\mathrm{B}$. thuringiensis.

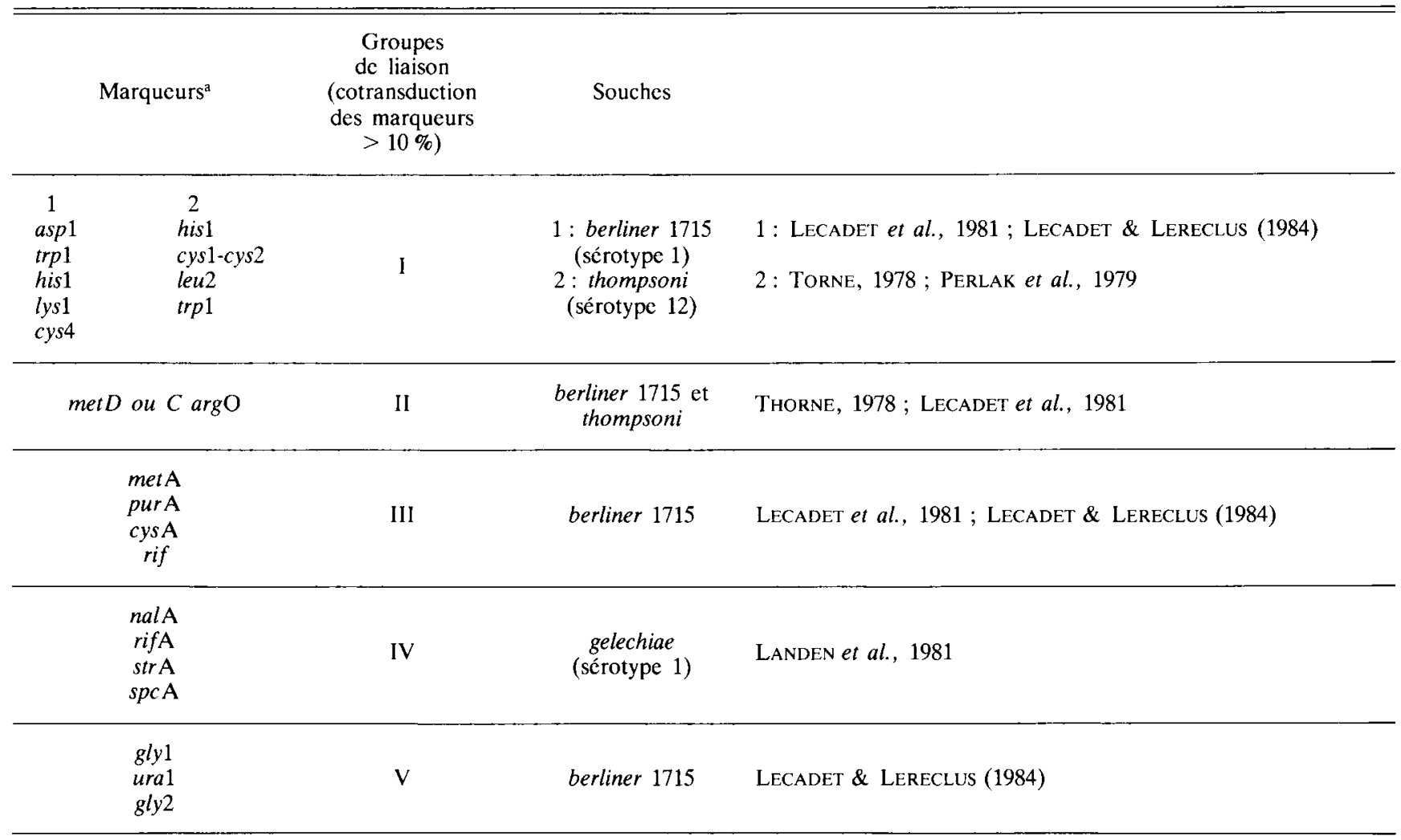

a : La nomenclature des marqueurs correspond à celle utilisée pour B. subtilis (YouNG \& WiLSON, 1975).

$a$ : The nomenclature of markers corresponds to those used for B. subtilis (YOUNG \& WILSON, 1975).

leur masse moléculaire. Ce point a été vérifié par les expériences d'hybridation moléculaire qui ont aussi indiqué que plusieurs plasmides, rassemblés en 2 catégories de taille ( $<$ et $>15 \mathrm{Md}$ ), étaient conservés, totalement ou partiellement, au travers des différents sérotypes. Ces premières observations qui ont abouti à établir 2 principaux groupes d'homologie parmi les plasmides, se sont avérées compatibles avec les résultats concernant la localisation du gène du cristal. Le clonage du gène ainsi que les expériences de conjugaison ont en effet montré que le gène codant pour la $\delta$-endotoxine était porté, dans la plupart des souches, par un plasmide appartenant au même groupe d'homologie (celui comprenant les plasmides de masse moléculaire élevée). Le tableau 4 rassemble tous les résultats concernant la localisation des gènes codant pour le cristal protéique, résultats obtenus soit par clonage et hybridation avec le gène cloné, soit par l'examen des schémas extrachromosomiques des souches $\mathrm{Cry}^{-}$isolées après avoir provoqué la perte du plasmide porteur du gène, soit enfin à l'issue d'expériences de conjugaison.

Si diverses situations sont donc possibles quant au déterminisme génétique du cristal protéique, la localisation du gène sur un plasmide de masse moléculaire supérieure à $30 \mathrm{Md}$ semble constituer un trait prédominant. En revanche, une localisation unique, sur le chromosome ou sur un plasmide de taille élevée ( $>150 \mathrm{Md}$ ), n'est observable que dans 2 souches : la souche dendrolimus (KLIER et al., 1982) et la souche entomocidus puisqu'elle ne semble pas posséder de plasmides. La liste des souches présentant une double localisation du gène n'est pas exhaustive, du fait que celle-ci n'a pas été recherchée dans tous les cas; néanmoins il apparaît nettement que le phénomène de double localisation n'est pas exceptionnel ; il s'applique en effet au moins aux 3 souches suivantes : berliner 1715, kurstaki et subtoxicus. Jusqu'à présent, les travaux réalisés sur ces isolats semblent indiquer qu'un seul des 2 gènes (d'origine chromosomique ou plasmidique) puisse s'exprimer après clonage chez $E$. coli ou $B$. subtilis. Les expériences destinées à provoquer la perte d'un plasmide semblent démontrer que, chez $B$. thuringiensis, seul le gène d'origine plasmidique est actif dans les conditions habituelles de croissance (à $30^{\circ} \mathrm{C}$ en présence de glucose). Cependant, on peut remarquer que, là encore, le manque de rigueur dans la désignation des souches (principalement au niveau des souches kurstaki) crée une certaine ambiguilé dans l'interprétation des résultats et ne permet pas de conclure d'une façon définitive sur ce point précis.

Pour en revenir à la localisation du gène du cristal, 2 autres résultats doivent être soulignés: ils concernent, d'une part, la souche kurstaki HD1 Dipel, où le gène serait présent à la fois sur 2 plasmides (tabl. 4) et, d'autre part, une souche israelensis, pour laquelle il est suggéré que le gène du cristal est porté par un plasmide de taille nettement inférieure, d'environ $4 \mathrm{Md}$ (FAUST et al., 1983). D'autres auteurs, au contraire, indiquent que dans cette souche le plasmide impliqué a une masse de $72 \mathrm{Md}$ (CARLTON et al., 1982).

Ces 2 observations, ajoutées aux divers types de localisation décrits précédemment, incitent à faire l'hypothèse que la répartition du gène du cristal dans les différents sérotypes 
TABLEAU 4

Localisation des gènes codant pour le cristal protéique.

Location of the genes coding for the crystal protein.

\begin{tabular}{|c|c|c|c|c|c|}
\hline H. sérotype & Souches & $\begin{array}{l}\text { Plasmides }^{\mathrm{a}} \\
\text { masse en Md }\end{array}$ & Chromosome & Modes de détermination & Références \\
\hline \multirow[t]{4}{*}{1} & thuringiensis $\mathrm{HD}-2$ & 75 & & Pertc provoquée, conjugaison & $\begin{array}{l}\text { GONZALEZ et al. (1981, } \\
\text { 1982) }\end{array}$ \\
\hline & thuringiensis berliner 1715 & 42 & & $\begin{array}{l}\text { Clonage et expression hétérospé- } \\
\text { cifique }\end{array}$ & KLIER et al. (1982) \\
\hline & & & + & $\begin{array}{l}\text { Hybridation avec le fragment } \\
\text { d'ADN cloné }\end{array}$ & KLIER et al., (1982) \\
\hline & thuringiensis berliner 22105 & 56 & & $\begin{array}{l}\text { Hybridation avec le fragment } \\
\text { d'ADN cloné }\end{array}$ & KLIER et al. (1982) \\
\hline \multirow[t]{2}{*}{$3 a$} & alesti $\mathrm{HD}-4$ & 105 & & Perte provoquéc & GONZALEZ et al. (1981) \\
\hline & alesti HD-4 & $>100$ & & $\begin{array}{l}\text { Hybridation avec le fragment } \\
\text { d'ADN cloné }\end{array}$ & WHITELEY et al. (1982) \\
\hline \multirow[t]{7}{*}{$3 a 3 b$} & kurstaki HD-1 (Dipel) & $32 \mathrm{et}>100$ & $?$ & $\begin{array}{l}\text { Clonage et expression hétérospé- } \\
\text { cifique }\end{array}$ & $\begin{array}{l}\text { SCHNEPF } \\
(1981)\end{array}$ \\
\hline & kurstaki HD-1 & 47 & - & $\begin{array}{l}\text { Hybridation avec le fragment } \\
\text { d'ADN cloné }\end{array}$ & KLIER et al. (1982) \\
\hline & kurstaki HD-1 & 110 & & Perte provoquéc & CARLTON et al. (1982) \\
\hline & kurstaki HD-73 & 50 & & Perte provoquće, conjugaison & $\begin{array}{l}\text { GONZALEZ et al. (1981, } \\
\text { 1982) }\end{array}$ \\
\hline & kurstaki HD-73 & 56 & & $\begin{array}{l}\text { Clonage et expression hétérospé- } \\
\text { cifique }\end{array}$ & WHITELEY et al. (1982) \\
\hline & kurstaki HD-263 & 44 & & Perte provoquéc, conjugaison & GoNZALEZ et al. (1982) \\
\hline & kurstaki souche? & 30 & $\begin{array}{l}\mathrm{C} \\
\mathrm{c} \\
\mathrm{i} \\
\mathrm{d}\end{array}$ & $\begin{array}{l}\text { Clonage et expression hétérospé- } \\
\text { cifique } \\
\text { Hybridation avec le fragment } \\
\text { d'ADN cloné }\end{array}$ & HELD et al. (1982) \\
\hline \multirow[t]{3}{*}{$4 a 4 b$} & sotto & 33 & & $\begin{array}{l}\text { Clonage et expression hétérospé- } \\
\text { cifique }\end{array}$ & WHITELEY et al. (1982) \\
\hline & sotto & 40 & & $\begin{array}{l}\text { Hybridation avec le fragment } \\
\text { d'ADN cloné }\end{array}$ & KLIER et al. (1982) \\
\hline & dendrolimus & - & + & $\begin{array}{l}\text { Hybridation avec le fragment } \\
\text { d'ADN cloné }\end{array}$ & KLIER et al. (1982) \\
\hline $5 \mathrm{a} 5 \mathrm{~b}$ & galleriae $\mathrm{HD}-8$ & $\approx 130$ & & Perte provoquéc & GONZALEZ et al. (1981) \\
\hline 6 & subtoxicus & 52 ou 56 & + & $\begin{array}{l}\text { Hybridation avec le fragment } \\
\text { d'ADN cloné }\end{array}$ & KLIER et al. (1982) \\
\hline \multirow[t]{2}{*}{9} & tolworthi & 40 à 60 & & $\begin{array}{l}\text { Hybridation avec le fragment } \\
\text { d'ADN cloné }\end{array}$ & KLIER et al. (1982) \\
\hline & tolworthi & 50 & & $\begin{array}{l}\text { Hybridation avec le fragment } \\
\text { d'ADN cloné }\end{array}$ & WHITELEY et al. (1982) \\
\hline 10 & darmstadiensis & 50 & & $\begin{array}{l}\text { Hybridation avec le fragment } \\
\text { d'ADN cloné }\end{array}$ & WHITELEY et al. (1982) \\
\hline $11 \mathrm{a} 11 \mathrm{~b}$ & toumanoffi & $>100$ & & $\begin{array}{l}\text { Hybridation avec le fragment } \\
\text { d'ADN cloné }\end{array}$ & WHITELEY et al. (1982) \\
\hline 14 & israelensis HD-567 & 72 & & Perte provoquéc, conjugaison & CARLTON et al. (1982) \\
\hline
\end{tabular}

Le signe + indique que le gène du cristal est présent sur le chromosome ou, comme le suggèrent KronSTAD et al. (1983), sur un plasmide de masse moléculaire élevée (>150 Md) non différencié de l'ADN chromosomique.

a : Cette colonne indique la masse moléculaire des plasmides porteurs du gène du cristal.

The sign + indicates that the crystal gene was present on the chromosome or, as suggested by KRONSTAD et al. (1983), on a high molecular weight plasmid ( $>150 \mathrm{Md}$ ) undistinguishable from the chromosomal DNA.

$a$ : This column indicates the molecular weight of plasmids harbouring the crystal gene.

de $B$. thuringiensis, et principalement à l'intérieur d'une même cellule, puisse avoir à son origine un mécanisme de transposition d'ADN faisant intervenir une structure moléculaire assimilable à une séquence d'insertion. Les travaux qui ont abouti à l'isolement, par transposition in vivo, d'une séquence d'ADN commune à plusieurs plasmides dont ceux porteurs du gène du cristal (LERECLUS et al., 1983) semblent aussi aller dans le sens d'une telle hypothèse. Sur ce sujet un travail intéressant peut être envisagé pour rechercher s'il existe une relation entre lc gène du cristal et une séquence d'insertion et de quel type de relation il s'agit.

D'une façon générale, l'ensemble des recherches réalisées sur la génétique de $B$. thuringiensis offre de larges perspectives tant sur le plan fondamental qu'au niveau appliqué.
L'exploitation des données actuelles sur le déterminisme de la synthèse du cristal protéique et l'utilisation du système de conjugaison convergeront en effet vraisemblablement vers une meilleure connaissance de l'expression et de la régulation du ou des gènes impliqués dans cette synthèse. Le cristal étant une protéine parasporale, c'est donc aussi sur le phénomène simple de différenciation cellulaire qu'est la sporulation que ces recherches pourront apporter des informations intéressantes. On peut bien entendu entrevoir d'autres développements à ces travaux, en particulier sur la fonction des plasmides de cet organisme.

En ce qui concerne les applications de ces recherches, hormis l'utilisation actuelle du cristal des souches berliner 1715 et kurstaki HD1 contre un nombre limité de 
lépitoptères, elles peuvent être réparties en 2 secteurs :

- tout d'abord celui représenté par le cristal protéique de la souche israelensis. La $\delta$-endotoxine produite par cette bactérie suscite en effet beaucoup d'intérêt du fait de son activité contre les larves de moustiques, vecteurs des parasites responsables du paludisme, de la fièvre jaune et de l'onchocercose. Le clonage du gène correspondant est entrepris dans de nombreux laboratoires et pourrait permettre, par association avec le gène codant pour les toxines produites par B. sphaericus (TINELLI et al., 1980 ; DAVIDSON, 1981), d'élargir le spectre d'activité de ces bactéries contre les moustiques.

- le $2^{\mathfrak{c}}$ secteur résultera de l'élargissement du spectre de toxicité de $B$. thuringiensis en vue d'étendre ses possibilités d'utilisation à d'autres espèces phytophages de grande importance économique à l'échelle mondiale et difficiles à combattre à l'aide des moyens chimiques actuellement disponibles. Il s'agit notamment de ravageurs polypilages appartenant à la famille des Noctuidae parmi lesquels il faut distinguer, en tout premier lieu, Spodoptera littoralis Boisd., ravageuse du coton et des légumincuses fourragères dans les régions circum-méditerranéennes, ainsi que Mamestra brassicae L. dont les dégâts intéressent un grand nombre de cultures basses en Europe.

En raison de son grand intérêt économique, ce sujet fait actuellement l'objet des préoccupations d'autres chercheurs dont les premiers travaux ont consisté à rechercher des bactéries naturellement actives contre ces insectes. Une souche aïzawai du sérotype 7 et une souche entomocidus du sérotype 6 ont ainsi été dégagées comme produisant un cristal relativement plus actif contre $S$. littoralis, la noctuelle du coton (LUTHY et al., 1982 ; SALAMA \& FODA, 1982). L'augmentation du niveau de toxicité du cristal de cette souche peut alors être envisagée, soit par des procédés biochimiques, soit par voie génétique. Sur ce dernier point, le clonage du gène correspondant pourrait être suivi d'expćriences de mutagenèse in vitro ou d'une association avec un autre gène dans une même bactérie, cela en vue d'obtenir l'augmentation du niveau de toxicité par modification du cristal ou par effet synergique.

Introduire dans une seule cellule 2 ou plusieurs gènes codant pour des cristaux protéiques dont les toxicités sont complémentaires reviendrait à construire une bactéric présentant un spectre d'activité plus large vis-à-vis des insectes. Il a été en effet récemment montré que l'association, par conjugaison, de 2 plasmides codant pour des cristaux distincts (spécifiques des sérotypes 1 et 3 ) aboutit à la synthèse de cristaux ayant les caractères antigéniques des 2 souches d'origine (GonZaLEZ et al., 1982).

En conclusion, si les importantes perspectives offertes par $B$. thuringiensis sur le plan agronomique ont largement contribué à l'évolution rapide des recherches d'ordre fondamental chez cet organisme et principalement sur le déterminisme de la $\delta$-endotoxine, les résultats obtenus laissent envisager à leur tour non seulement le développement des applications mais aussi celui des recherches menées en amont sur les caractéristiques génétiques de cettc bactéric.

Reçu le 6 avril 1983.

Accepté le 26 octobre 1983

\section{REMERCIEMENTS}

Je tiens à remercier Marguerite-M. LECADET et Daniel MARTOURET dont les connaissances sur le sujet ont bicn facilité l'élaboration de cette revue. Je suis aussi très reconnaissant envers Daniclle LEFEVRE pour son concours sympathique et efficace.

\section{RÉFÉRENCES BIBLIOGRAPHIQUES}

Alikhanian S. I., Ryabchenko N. F., Bukanov N. O., Sakanyan V. A., 1981. Transformation of Bacillus thuringiensis subsp. galleria protoplasts by plasmid pBC16. J. Bacteriol., 146, 7-9.

Angus T. A., Norris J. R., 1968. A comparison of the toxicity of some varieties of Bacillus thuringiensis Berliner for silkworm larvac. J. Invertebr. Pathol. 11, 289-295.

Barjac H. de, 1978. Toxicité de B. thuringiensis var. israelensis pour les larves d'Aedes aegypti et d'Anopheles stephensi. C. R. Acad. Sci. Paris, sér. D, 286, 1175-1178.

Barjac H. de, 1981. Identification of $\mathrm{H}$. serotype of Bacillus thuringiensis, p. 35-43. In: H. D. Burges. Microbial control of pest and plant diseases, 1970-1980. Academic Press Inc., Ncw York, 949 p.

Barjac H. de, Bonnefoi A., 1968. A classification of Bacillus thuringiensis Berliner with a key to their differentiation. J. Invertebr. Pathol., 11, 335-347.

Bernhard K., Schrempf H., Goebel W., 1978. Bacteriocin and antibiotic resistance plasmids in Bacillus cereus and Bacillus subtilis. J. Bacteriol., 133, 897-903.

Bulla L. A. Jr, Kramer K. J., Davidson L. T., 1977. Characterization of the entomocidal parasporal crystal of Bacillus thuringiensis. J. Bacteriol., 130, 373-383.

Bulla L. A. Jr, Davidson L. I., Kramer K. J., Jones B. L., 1979. Purification of the insecticidal toxins from the parasporal crystal of Bacillus thuringiensis subsp. kurstaki. Biochem. Biophys. Res. Commun., 91, 1123-1130.

Carlton B. C., Gonzalez J. M. Jr, Brown B. J., 1982. Assignment of delta-endotoxin genes of Bacillus thuringiensis to specific plasmids by curing and plasmid transfer analyses, p. 68. In : Abstr. IIIrd Int. Coll. Invertebr. Pathol., Brighton (U.K.).
Chang S., Cohen S. N., 1979. High frequency transformation of Bacillus subtilis protoplasts. Mol. Gen. Genet., 168, 111-115.

Clewell D. B., Yagi Y., Dunny C. M., Schultz S. K., 1974. Characterization of three plasmid deoxyribonucleic acid molecules in a strain of Streptococcus faecalis : identification of a plasmid determining erythromycin resistance. J. Bacteriol., 117, 283-289.

Davidson E. W., 1981. Bacterial diseases of insect caused by toxinproducing bacilli other than Bacillus thuringiensis. p. 269-291. In : E. W. Davidson. Pathogenesis of invertebrate microbial diseases. Allanheld, Osmun, Totowa, N. J. 562 p.

Debabov V. G., Azizbekyan R. R., Khlebalina O. I., D'Yachenko V. V., Galushka F. P., Belykh R. A., 1977. Isolation and preliminary characterization of extrachromosomal elements of Bacillus thuringiensis DNA. Genetika, 13, 496-501.

Farkas J., Sebesta K., Horska K., Samek Z., Dolijs J., Sorm F., 1969. The structure of exotoxin of Bacillus thuringiensis var. galechiae. Collect. Czech. Chem. Commun., 34, 1118-1120.

Faust R. M., Abe K., Held G. A., Iizuka T., Bulla L. A., Meyers C. L., 1983. Evidence for plasmid-associated crystal toxin production in Bacillus thuringiensis subsp. israelensis. Plasmid, 9, 98-103.

Glatron M.-F., Rapoport G., 1972. Biosynthesis of the parasporal inclusion of Bacillus thuringiensis : half-life of its corresponding messenger RNA. Biochimie, 54, 1291-1301.

Glatron M.-F., Lecadet M.-M., Dedonder R., 1972. Structure of the parasporal inclusion of Bacillus thuringiensis Berliner; characterization of a repetitive subunit. Eur. J. Biochem., 30, 330-338. Gonzalez J. M. Jr, Carlton B. C., 1980. Patterns of plasmid DNA in crystalliferous and acrystalliferous strains of Bacillus thuringiensis. Plasmid, 3, 92-98. 
Gonzalez J. M. Jr, Dulmage H. T., Cariton B. C., 1981. Correlation between specific plasmids and $\delta$-cndotoxin production in Bacillus thuringiensis. Plasmid, 5, 351-365.

Gonzalez J. M. Jr, Carlton B. C., 1982. Plasmid transfer in Bacillus thuringiensis. p. 85-95, In: Streips U. N., Goodgal S. H., Guild W. R., Wilson G. A. Genetic exchange : a celebration and $a$ new generation, Dekker, New York.

Gonzalez J. M. Jlr, Brown B. J., Carlton B. C., 1982. Transfer of Bacillus thuringiensis plasmids coding for $\delta$-endotoxin among strains of $B$. thuringiensis and B. cereus. Proc. Natl. Acad. Sci. U.S.A., 79, 6951-6955.

Held G. A., Bulla L. A. Jr, Ferrari E., Hoch J., Aronson A. I., Munnich S. A., 1982. Cloning and localization of the lepidopteran protoxin gene of Bacillus thuringiensis subsp. kurstaki. Proc. Natl. Acad. Sci. U.S.A., 79, 6065-6069.

Klier A., Lecadet M.-M., Rapoport G., 1978. Transcription in vitro of sporulation-specific mRNAs by RNA polymerase from Bacillus thuringiensis, p. 205-211. In : Chambliss G. Vary J. C. Spore VII, Am. Soc. Microbiol., Wash. D.C., 354 p.

Klier A. F., Kunst F., Rapoport G., 1979. Structure of cloned ribosomal DNA cistrons from Bacillus thuringiensis. Nucl. Acids Res., 7, 997-1010.

Klier A., Fargette F., Ribier J., Rapoport G., 1982. Cloning and expression of the crystal protein genes from Bacillus thuringiensis strain berliner 1715. EMBO J., 1, n 7, 791-799.

Klier A., Parsot C., Rapoport G., 1983. In vitro transcription of the cloned chromosomal crystal gene from Bacillus thuringiensis. Nucl. Acids Res., 11, 3973-3987.

Kronstad J. W., Schnepf H. E., Whiteley H. R., 1983. Diversity of locations for Bacillus thuringiensis crystal protein genes. J. Bacteriol., 154, 419-428.

Landen R., Heierson A., Boman H. G., 1981. A phage for generalized transduction in Bacillus thuringiensis and mapping of four genes for antibiotic resistance. J. Gen. Microbiol, 123, 49-59.

Lecadet M.-M., 1970. Bacillus thuringiensis toxins. The proteinaceous crystal, p. 4.37-471. In : Montie T. C., Kadis S., Aijl S. J., Microbial toxins, vol. 3, Academic Press Inc., New York., 548 p. Lecadet M.-M., Martouret D., 1962. La toxinc figurée de $B$. thuringiensis: production enzymatique de substances solubles toxiques par injection. C.R. Acad. Sci. Paris, Sér. D, 254, 2457-2460. Lecadet M.-M., Martouret D., 1967. Enzymatic hydrolysis of the crystal of $B$. thuringiensis by the proteases of Pieris brassicae. II. Toxicity of the different fractions of the hydrolysate for larvae of Pieris brassicae. J. Invertebr. Pathol., 2, 322-330.

Lecadet M.-M., Blondel M.-O., Ribier J., 1980. Generalized transduction in Bacillus thuringiensis var. berliner 1715, using bacteriophage CP54 Ber. J. Gen. Microbiol., 121, 203-212.

Lecadet M.-M., Barjac H. de, 1981. Bacillus thuringiensis betaexotoxin, p. 293-321. In : Davidson F. W., Pathogenesis of invertebrate microbial diveases. Allanheld, Osmun. Totowa, N.J., 562 p.

Lecadet M.-M., Lereclus D., Blondel M.-O., Ribier J., 1981. Bacillus thuringiensis: studies on chromosomal and extrachromosomal DNA, p. 88-92. In: Levinson H. S., Sonenshein A. L., Tipper D. J., Sporulation and germination. Am. Soc. Microbiol., Wash. D.C., 317 p.

Lecadet M.-M., Lereclus D., 1984. Structure and activity of the $B$. thuringiensis $\delta$-endotoxin. Recent development in genetics. In : Bacterial proteins ioxins, F.E.M.S. Symposium 1983 (sous presse). Lereclus D., 1981. Etude des plasmides de Bacillus thuringiensis et préliminaires à l'élaboration d'un système de transformation chez cette bactérie. Thèse de $3^{\mathrm{e}}$ cycle. Univ. Paris VII, $102 \mathrm{p}$.
Lereclus D., Lecadet M.-M., Ribier J., Dedonder R., 1982. Molecular relationships among plasmids of Bacillus thuringiensis : conserved sequences through 11 crystalliferous strains. Mol. Gen. Genet., 186, 391-398.

Lereclus D., Menou G., Lecadet M.-M., 1983. Isolation of a DNA sequence related to several plasmids from Bacillus thuringiensis after a mating involving the Streptococcus faecalis plasmid pAMB1. Mol. Gen. Genet., 191, 307-313.

Luthy P., Ebersold H. R., 1981. The entomocidal toxins of $B$. thuringiensis, Pharm. Ther., vol. 13, 257-283. Pergamon Press Ltd. Luthy P., Jaquet F., Huber-Lukac H. E., Huber-Lukac M., 1982. Physiology of the delta-endotoxin of Bacillus thuringiensis including the ultrastructure and histopathological studies. p. 29-36. In : Basic biology of microbial larvicides of vectors of human diseases. W.H.O., V.B.C., Genève, 188 p.

Martin P. A. W., Lohr J. R., Dean D. H., 1981. Transformation of Bacillus thuringiensis protoplasts by plasmid deoxyribonucleic acid. J. Bacteriol., 145, 980-983.

Martouret D., 1978. Emplois de B. thuringiensis. Bull. Tech. Inf. Minist. Agric., 332-333, 465-470.

Miteva V. I., Shivarova N. I., Grigorova R. T., 1981. Transformation of Bacillus thuringiensis protoplasts by plasmid DNA F.E.M.S. Microbiol. Lett., 12, 253-256.

Perlak F. J., Mendelsohn C. L., Thorne C. B., 1979. Converting bacteriophage for sporulation and crystal formation in Bacillus thuringiensis. J. Bacteriol., 140, 699-706.

Salama H. S., Foda M. S., 1982. A strain of Bacillus thuringiensis var. entomocidus with high potential activity on Spodoptera littoralis. J. Invertebr. Pathol., 39, 110-111.

Schnepf H. E., Whiteley H. R., 1981. Cloning and expression of the Bacillus thuringiensis crystal protein gene in Escherichia coli, Proc. Natl. Acad. Sci. U.S.A., 78, 2893-2897.

Sebesta K., Horska K., 1970. Mechanisms of inhibition of DNA dependent RNA polymerase by exotoxin of Bacillus thuringiensis. Biochem. Biophys. Acta, 209, 357-376.

Stahly D. P., Dingman D. W., Bulla L. A. Jr, Aronson A. I., 1978. Possible origin and function of the parasporal crystals in Bacillus thuringiensis. Biochem. Biophys. Res. Comm., 84, 581-588.

Thorne C. B., 1978. Transduction in Bacillus thuringiensis. Appl. Environ. Microbiol., 38, 1109-1115.

Tinelli R., Barjac H. de, Bourgouin C., 1980. Isolement d'une fraction de spore de Bacillus sphaericus toxique pour les larves d'anophèles. C.R. Acad. Sci. Paris, Sér. D, 291, 537-539.

Tyrell D. J., Bulla L. A. Jr, Andrews R. E., Kramer K. J., Davidson L. I., Nordin P., 1981. Comparative biochemistry of entomocidal parasporal crystals of selected Bacillus thuringiensis strains. $J$. Bacteriol., 145, 1052-1062.

Whiteley H. R., Kronstad J. W., Schnepf H. E., 1982. Cloning the delta toxin gene of Bacillus thuringiensis in Escherichia coli and localization of the genc in different strains of $B$. thuringiensis, p. 147-156 In : Basic biology of microbial larvicides of vectors of human diseases. W.H.O., V.B.C., Genève, 188 p.

Wong H. C., Schnepf H. E., Whiteley H. R., 1983. Transcriptional and translational start sites for the Bacillus thuringiensis crystal protein gene. J. Biol. Chem., 258, 1960-1967.

Young F. E., Wilson G. A., 1975. Chromosomal map of Bacillus subtilis, p. 596-614. In : P. Gerhardt, R. N. Costilow, H. L. Sadoff. Spore VI. Am. Soc. Microbiol., Wash. D.C., 619 p. 